\title{
GEODESICS AND BOUNDED HARMONIC FUNCTIONS ON INFINITE PLANAR GRAPHS
}

\author{
S. NORTHSHIELD
}

(Communicated by William D. Sudderth)

\begin{abstract}
It is shown there that an infinite connected planar graph with a uniform upper bound on vertex degree and rapidly decreasing Green's function (relative to the simple random walk) has infinitely many pairwise finitelyintersecting geodesic rays starting at each vertex. We then demonstrate the existence of nonconstant bounded harmonic functions on the graph.
\end{abstract}

Let $\mathfrak{g}$ be an infinite, simple, connected, planar graph. $\mathfrak{g}$ also denotes the vertex set of the graph. If two vertices $x$ and $y$ are connected by an edge, we write $x E y$. For a vertex $x$, the degree of $x$ is $d(x) \equiv|\{y \in \mathfrak{g}: y E x\}|$, and we assume:

$$
\delta \equiv \sup _{x \in \mathfrak{g}} d(x)<\infty .
$$

A finite [infinite] walk $\gamma$ is a sequence $(\gamma(0), \ldots, \gamma(n))[(\gamma(0), \gamma(1), \ldots)]$ of elements of $\mathfrak{g}$ such that $\gamma(k) E \gamma(k+1)$ for all $0 \leq k \leq n-1$ [for all $k \geq 0$ ]. We say that $\gamma$ starts at $\gamma(0)$ and, in the first case, ends at $\gamma(n)$ and has length $n$. Since $\mathfrak{g}$ is connected, we may define a metric:

$$
d(x, y) \equiv \inf \{n: n \text { is the length of a finite walk from } x \text { to } y\} .
$$

A path is a walk whose vertices are distinct. A geodesic $\gamma$ is a path such that $d(\gamma(m), \gamma(n))=|m-n|$ for all possible $m$ and $n$. For $x \in \mathfrak{g}, \Gamma(x, n)$ is the set of geodesics that have length $n$ and start at $x ; \Gamma(x)$ is the set of geodesics that have infinite length and start at $x$.

The following propositions are useful; the first is easy to prove by a diagonal type argument.

Proposition 1. For all $x \in \mathfrak{g}, \Gamma(x) \neq \varnothing$.

Proposition 2. Given $x, y \in \mathfrak{g}$ and $\gamma \in \Gamma(x)$, there exists a $\gamma^{\prime} \in \Gamma(y)$ such that $\gamma$ and $\gamma^{\prime}$ eventually coincide.

Received by the editors October 10, 1989 and, in revised form, September 26, 1990.

1980 Mathematics Subject Classification (1985 Revision). Primary 60J45; Secondary 60J15, $05 \mathrm{C} 38$.

Key words and phrases. Random walk, planar graphs, geodesic rays, harmonic functions.

During this research, the author was supported by a graduate fellowship at the University of Rochester. 
Proof. Let $x, y \in \mathfrak{g}, \gamma \in \Gamma(x)$. By the triangle inequality, $|d(y, \gamma(n))-n|=$ $|d(y, \gamma(n))-d(x, \gamma(n))| \leq d(x, y)$ and, since $d(y, \gamma(n))-n$ is nonincreasing, $a \equiv \lim _{n \rightarrow \infty}[d(y, \gamma(n))-n]=d(y, \gamma(N))-N$ for some $N$. Define a path $\gamma^{\prime}$ where $\left(\gamma^{\prime}(0), \ldots, \gamma^{\prime}(d(y, \gamma(N)))\right)$ is a finite geodesic from $y$ to $\gamma(N)$ and, for $k \geq d(y, \gamma(N)), \gamma^{\prime}(k)=\gamma(k-a)$. Then $\gamma^{\prime} \in \Gamma(y)$.

Consider the transition probabilities for a Markov chain defined by:

$$
p(x, y) \equiv \begin{cases}1 / d(x) & \text { if } y E x \\ 0 & \text { otherwise }\end{cases}
$$

We denote this chain by $X(0), X(1), \ldots$ We let $P^{x}(\cdot) \equiv P(\cdot \mid X(0)=x)$ and $E^{x}(\cdot)$ be the associated expectation operator. Hence, $p(x, y)=P(X(1)=$ $y \mid X(0)=x)=P^{x}(X(1)=y) . X(\cdot)$ is called the simple random walk on $\mathfrak{g}$.

Let $p^{(n)}(x, y)$ be the $n$-fold convolution of $p$ with itself, and define Green's function as $G(x, y)=\sum_{n \geq 0} p^{(n)}(x, y)$. Probabilistically, $p^{(n)}(x, y)=P^{x}(X(n)$ $=y)$ and $G(x, y)=E^{x}\left(\sum_{n \geq 0} \chi_{\{y\}}(X(n))\right)=$ the average number of times that the random walk, starting at $x$, hits $y$. It is easy to see that the random walk is transient if and only if $G$ exists (see [2]; his proof for the case when $\mathfrak{g}$ is a tree applies to our case without change). By the strong Markov property,

$$
G(x, y)=P^{x}(\exists n \geq 0: X(n)=y) G(y, y)
$$

We assume that Green's function is rapidly decreasing in the sense that

$$
\sum_{n \geq 0} n \cdot \sup \{G(x, y): x, y \in \mathfrak{g}, d(x, y)=n\}<\infty
$$

Remark. It is known that the Cheeger condition

$$
\exists c>0: \forall \text { finite } K \subset \mathfrak{g}: \#\left\{\text { edges from } K \text { to } K^{c}\right\} /|K| \geq c
$$

implies $G(x, y) \leq c \varepsilon^{d(x, y)}$ (for some $c$ and $\varepsilon$ )-see [1] or [4]. Hence the Cheeger condition implies condition (3).

Lemma 1. For any integer $m \geq 0$, there is an $N(m) \geq 0$ such that if $A$ is the union of $m$ geodesics and $d(x, A) \geq N(m)$, then $P^{x}(\exists n: X(n) \in A)<1$.

Proof. For any $n \geq 0, x \in \mathfrak{g}$, let $S(x, n)$ and $B(x, n)$ be the metric sphere and ball respectively with centers $x$ and radii $n$. If $\gamma$ is a geodesic, then $|\gamma \cap S(x, n)| \leq|\gamma \cap B(x, n)| \leq 2 n+1$. Hence $|A \cap S(x, n)| \leq(2 n+1) m$ and 
we get

$$
\begin{aligned}
P^{x}(\exists n \geq 0: X(n) \in A) & \leq \sum_{y \in A} P^{x}(\exists n \geq 0: X(n)=y) \\
& =\sum_{y \in A} \frac{G(x, y)}{G(y, y)} \quad(\text { by }(2)) \\
& \leq \sum_{y \in A} G(x, y) \quad(\text { since } G(y, y) \geq 1) \\
& \leq \sum_{n \geq d(x, A)}|A \cap S(x, n)| \cdot \sup \{G(x, y): d(x, y)=n\} \\
& \leq m \sum_{n \geq d(x, A)}(2 n+1) \cdot \sup \{G(x, y): d(x, y)=n\}
\end{aligned}
$$

By (3), choose $N(m)$ so that $m \sum_{n \geq N(m)}(2 n+1) \cdot \sup \{G(x, y): d(x, y)=n\}<$ 1 .

Lemma 2. For any $K \subset \mathfrak{g}$, if $\inf _{x \in \mathfrak{g}} P^{x}\left(\limsup _{n \rightarrow \infty}(X(n) \in K)\right)<1$, then $\sup _{x \in \mathfrak{g}} d(x, K)=\infty$.

Proof. By condition (1), for any $y \in K$ and $x \in \mathfrak{g}$,

$$
P^{x}(\exists n: X(n) \in K) \geq P^{x}(X(d(x, y))=y) \geq(1 / \delta)^{d(x, y)} .
$$

Thus, if $\sup _{x \in \mathfrak{g}} d(x, K)<\infty$, then $\inf _{x \in \mathfrak{g}} P^{x}(\exists n: X(n) \in K)>0$ and, therefore, $\inf _{x \in \mathfrak{g}} P^{x}\left(\lim \sup _{n \rightarrow \infty}(X(n) \in K)\right)=1$.

Theorem 1. For any $x \in \mathfrak{g}$, there are infinitely many geodesic rays $\gamma_{1}, \gamma_{2}, \ldots$ starting at $x$ such that if $i \neq j$, then $\left|\gamma_{i} \cap \gamma_{j}\right|<\infty$.

Proof. We construct such a family inductively. There is always one geodesic ray starting at $x$ (Proposition 1). Suppose $\gamma_{1}, \gamma_{2}, \ldots, \gamma_{m} \in \Gamma(x)$ such that if $i \neq j$, then $\left|\gamma_{i} \cap \gamma_{j}\right|<\infty$. Let $\partial A=\bigcup_{i=1}^{n} \gamma_{i}$. By Proposition 2, it is enough to show that there exists a geodesic ray $\gamma$ such that $\gamma \cap \partial A=\varnothing$. Therefore, by the diagonal method of Proposition 1, it is enough to show that there exists $z \in \mathfrak{g}$ such that for all $k$, there exists $\gamma_{k} \in \Gamma(z, k)$ so that $\gamma_{k} \cap \partial A=\varnothing$.

Let $A=\mathfrak{g} \backslash \partial A$ and $N=N(m+2)$ where $N(\cdot)$ is as in Lemma 1 . As in the proof of Lemma 1, $\sum_{y \in \partial A} G(x, y)<\infty$ and so

$$
P^{x}\left(\limsup _{k \rightarrow \infty}(X(k) \in \partial A)\right)=0 .
$$

By Lemma 2, we can choose $z \in A$ such that $d(z, \partial A) \geq N$.

Suppose that there exists $n$ such that for all $\gamma \in \Gamma(z, n), \gamma \cap \partial A \neq \varnothing$. We show that this leads to a contradiction-we show that this implies the existence of two geodesic segments $\gamma_{t}^{*}$ and $\gamma_{u}^{*}$ such that:

(a) $d\left(z, \gamma_{t}^{*} \cup \gamma_{u}^{*}\right) \geq N$ and

(b) every infinite path starting at $z$ hits $\gamma_{t}^{*} \cup \gamma_{u}^{*} \cup \partial A$.

By Lemma 1, condition (a) implies $P^{z}\left(\exists j: X(j) \in \gamma_{t}^{*} \cup \gamma_{u}^{*} \cup \partial A\right)<1$ whereas condition (b) implies $P^{z}\left(\exists j: X(j) \in \gamma_{t}^{*} \cup \gamma_{u}^{*} \cup \partial A\right)=1$. 
For each $y \in S(z, n)$, choose $\gamma_{y} \in \Gamma(z, n)$. In addition, we choose these geodesics so that $\bigcup \gamma_{y}$ is a tree. For any $y \in S(z, n)$, let $\gamma_{y}^{*}=\left(\gamma_{y}(\eta), \ldots, \gamma_{y}(n)\right)$ where $\eta=\max \left\{j \leq n: \gamma_{y}(j) \in \partial A\right\}$. Note that for any $t, u \in S(z, n)$, condition (a) holds. Let $Z=\{y \in S(z, n)$ : there exists an infinite path in $A$, starting at $z$, which last hits $B(z, n)$ at $y\} . Z$ is nonempty by choice of $N$ and $z$. For $Y \subset Z$, let $C(Y)$ be the connected component of $B(z, n) \backslash$ $\left(\partial A \cup \bigcup_{y \in Y} \gamma_{y}^{*}\right)$ which contains $z$.

We claim that $C(Z)=C(\{t, u\})$ for some $t, u \in Z$. If so, then condition (b) holds for $t$ and $u$. To prove this claim, it is enough to show that if $t, u$, $v$ are distinct elements of $Z$, then $C(\{t, u, v\})=C\left(\left\{\dot{t}^{\circ}, u^{\circ}\right\}\right)$ for some $t^{\circ}$, $u \in\{t, u, v\}$.

Let $t, u, v$ be distinct elements of $Z$, and let $\rho, \sigma, \tau$ be infinite paths in $A$ starting at $z$ which last hit $B(z, n)$ at $t, u, v$ respectively. Since $\partial A$ is connected, $\partial A$ is in one of the components of $G \backslash(\rho \cup \sigma \cup \tau)$. By planarity, without loss of generality, any path from $t$ to $\partial A$ must hit $\sigma \cup \tau$. Define $\rho^{*}(j)=\rho(j+M+1)$ where $M=\max \{k: \rho(k) \in B(z, n)\}$. Then the complement of $\partial A \cup \gamma_{t}^{*} \cup \rho^{*}$ contains two components, say $B$ and $C$, such that $u \in B, v \in C$, and, without loss of generality, $z \in B$. Then, any path contained in $A$ from $z$ to $v$ must hit either $\gamma_{t}^{*}$ or $\rho^{*}$. Since $\rho^{*} \cap B(z, n)=\varnothing$, $C(\{t, u, v\})=C(\{t, u\})$.

A function $f: \mathfrak{g} \rightarrow \mathbb{R}$ is harmonic if and only if $\sum_{y: y E x} f(y)=d(x) f(x)$ for all $x$. In particular, since $\liminf _{k \rightarrow \infty}(X(k) \in A)$ is invariant under the Markov shift, $f(x) \equiv P^{x}\left(\liminf _{k \rightarrow \infty}(X(k) \in A)\right)=p f(x)$ and so $f$ is bounded and harmonic. We use an idea similar to one Kendall uses in the case of Brownian motion on manifolds [3] to find a set $A$ so that $P^{*}\left(\liminf _{k \rightarrow \infty}(X(k) \in A)\right)$ is nonconstant.

Theorem 2. There are nonconstant, bounded, harmonic functions on $\mathfrak{g}$.

Proof. Let $N=N(2)$ where $N(\cdot)$ is as in Lemma 1. Fix $x \in \mathfrak{g}$ and, by Theorem 1 , choose $4 N$ rays $\gamma_{1}, \gamma_{2}, \ldots, \gamma_{4 N} \in \Gamma(x)$ whose pairwise intersections are finite. Without loss of generality, these geodesics are numbered in a clockwise fashion (we may do this since $\mathfrak{g}$ is planar). Let $M$ be such that $i \neq j$ implies $\left(\gamma_{i} \cap \gamma_{j}\right) \backslash B(x, M)=\varnothing$. Let $C=\gamma_{1} \cup \gamma_{2 N}, u=\gamma_{N}(M+N), v=\gamma_{3 N}(M+N)$, and $A$ and $B$ be the connected components of $\mathfrak{g} \backslash C$ containing $u$ and $v$ respectively. By Lemma 1 , since $d(u, C) \geq N$ and $d(v, C) \geq N$,

$$
P^{u}\left(\liminf _{k \rightarrow \infty}(X(k) \in A)\right) \geq P^{u}(\forall j: X(j) \notin C)>0
$$

and

$$
P^{v}\left(\limsup _{k \rightarrow \infty}(X(k) \in A)\right) \leq P^{v}(\exists j: X(j) \in C)<1 .
$$

By Lemma 2,

$$
\sup _{w \in \mathfrak{g}} d(w, A)=\infty
$$


Since, for $w \in B$,

$$
\begin{aligned}
P^{w}\left(\liminf _{k \rightarrow \infty}(X(k) \in A)\right) & \leq P^{w}(\exists j: X(j) \in C) \\
& \leq 2 c \sum_{n \geq d(w, A)}(2 n+1) \cdot \sup \{G(x, y): d(x, y)=n\}
\end{aligned}
$$

(as in the proof of Lemma 1), and since $d(w, A)$ is unbounded,

$$
\inf _{w} P^{w}\left(\liminf _{n \rightarrow \infty}(X(n) \in A)\right)=0
$$

and so is not constant.

\section{ACKNOWLEDGMENTS}

I would like to thank Mike Cranston for suggesting this problem, Carl Mueller for some useful advice, and the referee for suggesting the present form of (3).

\section{REFERENCES}

1. A. Ancona, Positive harmonic functions and hyperbolicity, Potential Theory (Kràl et al.) Lecture Notes in Math., vol. 1344, Springer-Verlag, Berlin, 1988, pp. 1-23.

2. P. Cartier, Fonctions harmoniques sur un Arbre, Symposia Mathematica, vol. 9, Academic Press, New York, 1982, pp. 203-270.

3. W. S. Kendall, Brownian motion on a surface of negative curvature, Séminaire de Probabililités 18, 1982/1983 (J. Azéma and M. Yor, eds.), Lecture Notes in Math., vol. 1059, Springer-Verlag, Berlin, 1984, pp. 70-76.

4. S. Northshield, Schrödinger operators on infinite graphs, doctoral dissertation, Univ. of Rochester, 1989.

Department of Mathematics, State University of New York College at Plattsburgh, Plattsburgh, New York 12901 